\title{
AN ADAPTIVE PROPORTIONAL-DERIVATIVE CONTROL METHOD FOR ROBOT MANIPULATOR
}

\author{
MAI THANG LONG, TRAN HUU TOAN, TRAN VAN HUNG, TRAN NGOC ANH, \\ NGUYEN HOANG HIEU \\ Faculty of Electronics Technology, Industrial University of Ho Chi Minh City \\ maithanglong@iuh.edu.vn
}

\begin{abstract}
This research presents an improved control method for the robot manipulator system based on the proportional-derivative technique and neural networks. In the proposed strategy, the proportionalderivative controller based on the filtered tracking error technique has been modified such that the proportional-derivative gain parameters are adaptively updated. Similar to the conventional intelligent control methods, the neural networks approximator is applied to relax the unknown dynamics of the robot control system. In addition, the compensator-typed robust controller is also considered to eliminate inevitable approximating errors and unknown disturbances of the control system. By using the Lyapunov stability theorem for the proposed control design procedure, the tracking control and stability are guaranteed. The comparative simulation results will provide clearly the evident to prove effectiveness of the proposed approach.
\end{abstract}

Keywords. Robot manipulators, PD/PID control, adaptive control, intelligent control.

\section{INTRODUCTION}

In fact, the robot manipulator (RM) control still always attracts attention from researchers to more improve tracking position control performance for industrial applications. In recent years, there are many intelligent control methods that have been explored to guarantee the RM control systems can be able to gain more effectiveness in stability, adaptation/flexibility and robustness features [1 - 16]. The authors in [3] provided the intelligent control methods for the RM system based on the adaptive neural networks (NN) to solve the uncertain RM dynamics and constraint on the joint positions in the requirement of well tracking errors performance. And also, by applying the NN, Zhou et al. [7] presented the control strategy for the RM system with dead zone, in which, the proportional-derivative (PD) controller based on the filtered tracking error technique and backstepping method were combined. However, the uncertain problems of the RM control system [7] have not addressed carefully yet. In the other hand, the authors in [9, 16] considered the nonsingular terminal sliding mode control schemes for the RM that achieved good performances in fast transient response and finite-time convergence. In general, the intelligent control methods in $[1-16]$ have a well-known property about the controller structure that can be review for improving the RM tracking position control. That is, in the structure of controllers [1 - 16], the proportional - integral - derivative (PID) technique (by using the proportional (P) part, or PD, or the proportional - integral (PI) parts, or PID parts) always plays an important role in forcing the position tracking errors to zero. Therefore, when considering to the filtered tracking errors methods typed PD controller $[1-16]$ we can easy realize the fixed PD gain problem that is a drawback. The tracking errors will decrease with increasing the PD gains. However, by adjusting to increase the PD gains for the desired tracking errors, the transient performance and stability of controlled system will be seriously affected if we cannot gain the optimal PD gains.

In order to solve the fixed PD gains problem to improve the tracking errors and stability performances, this study will propose a novel approach for the RM control as follows. The first, the PD controller based on the filtered tracking error technique will apply for the RM position control. The drawbacks of fixed PD gains will be relaxed by adaptive self-updating PD gain parameter in considering of the stability of the controlled system. The second, as similar to the intelligent control methods $[1-16]$, the unknown dynamics of the RM control system will be approximated by the adaptive NN approximator. The third, the tracking errors and robustness effectiveness will be more improved by the compensator-typed robust controller. This robust controller is designed to eliminate the $\mathrm{NN}$ approximating errors, the disturbances and uncertainties from the RM control system. In addition, the online learning/updating algorithms of control parameters in the proposed controller are designed based on the Lyapunov stability theorem such that the stability is 
guaranteed and the tracking errors will converge to zero as time tends to infinity.

This paper structure is arranged as follows. The part 2 reviews the dynamics, problem formulation and the applied NN model. The adaptive PD control algorithms are designed, analyzed and proved in the part 3 . In the part 4, the comparative simulation results for RM are demonstrated to prove the effectiveness of the proposed method. Finally, conclusions are drawn in the part 5.

\section{PRELIMINARIES}

\subsection{Problem formulation}

The dynamics of $n$-links robot manipulator system can be considered by the following Lagrange function form [1]:

$$
M(q) \ddot{q}+C(q, \dot{q}) \dot{q}+G(q)+F(\dot{q})+d=\tau
$$

where the $q, \dot{q}$ and $\ddot{q}$ are the joint position vector, velocity vector and acceleration vector, respectively. The $M(q) \in R^{n \times n}$ is the inertia matrix, the $C(q, \dot{q}) \dot{q} \in R^{n \times 1}$ is the vector of the centripetal and Coriolis forces. The $G(q) \in R^{n \times 1}, F(\dot{q}) \in R^{n \times 1}, \tau_{d} \in R^{n \times 1}$ and $\tau \in R^{n \times 1}$ are the gravity vector, friction vector, the unknown disturbances vector and the torque inputs vector, respectively. The following properties of the RM system are considered as [1]:

Property 1 . The $M(q)$ is a positive definite symmetric matrix and it is uniformly bounded as:

$$
m_{o 1}\|x\|^{2} \leq x^{T} M(q) x \leq m_{o 2}\|x\|^{2}, \forall x \in R^{n \times 1}
$$

where $m_{o 1}, m_{o 2}$ are the positive constants.

Property 2. Skew symmetric matrix, $S_{q}(q, \dot{q})$ is a skew symmetric matrix:

$$
S_{q} \equiv \dot{M}-2 C
$$

In this study, our purpose is to improve the traditional PD tracking control effectiveness for RM. So, based on the model of RM (1), we firstly define the tracking errors $e(t)$ and filtered-tracking errors $r(t)$ as the following forms $[1,17]$ :

$$
\left[\begin{array}{l}
e(t)=q_{d}-q \\
r(t)=\dot{e}+K_{r} e
\end{array}\right.
$$

where $q_{d}(t)$ is the smooth desired trajectory and it has derivative up to second order. $K_{r}=\operatorname{diag}\left(K_{r 1}, \ldots\right.$, ,$\left.K_{r n}\right)$ is positive constant matrix. The conventional control input can be applied as [1,17]:

$$
\tau^{*}=f+d+K_{p r} r
$$

where the function $\mathrm{f}$ is defined as $f=M\left(\ddot{q}_{d}+K_{r} \dot{e}\right)+C\left(\dot{q}_{d}+K_{r} e\right)+G+F$. The $K_{p r}=\operatorname{diag}\left(K_{p r 1}, \ldots, K_{p r n}\right)$ is the positive constant matrix. By the assumption that the dynamics of RM control system is known, the control input in (2) can guarantee the stability of the controlled system and the exponential convergence of the filtered-tracking errors [17]. However, the performance of tracking errors depends heavily on the selection of PD gains, $K_{p r}$ and $K_{r}$. In order to guarantee the requirement of filtered-tracking errors, the $K_{p r}$ and $K_{r}$ must be increased. We can see that if the proportional gains are too high, the controlled system can become unstable. In addition, with PD typed-filtered error, we difficult to adjust the proportional gain $K_{p r}$ and derivative gain $K_{r}$, separately. Therefore, this control input is not easy to apply in real control application. These problems will be addressed clearly in the next Sections.

\subsection{The NN approximator}

In this research, an adaptive NN approximator based on radial basic function neural networks (RBFNN) will be used to deal with the problem of unknown knowledge of the RM dynamics. The NN structure includes an input layer, a hidden layer and an output layer. The $\mathrm{NN}$ output layer can be described as following form [17]:

$$
f(u)=\mathrm{W}^{T} \phi(u)
$$

where $f=\left[f_{1}, f_{2}, \ldots, f_{m}\right], m \in N$ is the number of NN outputs. $\mathrm{W}^{T}=\left[\mathrm{w}_{1}, \mathrm{w}_{2}, \ldots, \mathrm{w}_{h}\right] \in R^{m \times h}$ is the weight matrix between the hidden layer and the output layer. $n, h \in N$ are the number of inputs, hidden nodes, 
respectively. $u=\left[u_{1}, u_{2}, \ldots, u_{n}\right]^{T}$ is the $\mathrm{NN}$ input. $\phi()=.\left[\phi_{1}, \phi_{2}, \ldots, \phi_{h}\right]^{T}$ is chosen as the Gaussian function, which is presented as the following form:

$$
\phi_{j}=e^{-\frac{\left(\left\|u-\mu_{i j}\right\|\right)^{2}}{2 \eta_{i j}^{2}}}
$$

with $i=1, \ldots, n, j=1, \ldots, h, \mu_{i j}=\left[\mu_{i 1}, \mu_{i 2}, \ldots, \mu_{i h}\right]^{T}$ and $\eta_{i j}=\left[\eta_{i 1}, \eta_{i 2}, \ldots, \eta_{i h}\right]^{T}$ are the center point and width of the Gaussian function. Based on the universal approximation capability of the NN [17], there exists an ideal NN structure as:

$$
f=\mathrm{W}^{* T} \phi(u)+\varepsilon(u)
$$

where $\mathrm{W}^{*}$ are the unknown optimal parameter, $\varepsilon(u)$ is the $\mathrm{NN}$ approximating error vector. Considering the approximation output of $\mathrm{NN}$ as:

$$
\hat{f}=\hat{\mathrm{W}}^{T} \phi(u)
$$

where $\hat{\mathrm{W}}$ is the approximation of the $\mathrm{W}^{*}$. Here, the inevitable error in the approximating process can be shown as follows:

$$
\tilde{f}=f-\hat{f}=\left(\mathrm{W}^{* T}-\hat{\mathrm{W}}^{T}\right) \phi+\varepsilon(u)=\tilde{\mathrm{W}}^{T} \phi+\varepsilon(u)
$$

In order to guarantee the control performance, the inevitable errors of the $\mathrm{NN}$ approximating process will be considered to eliminate. In addition, the NN weight parameter will also be adaptive updated to improve the adaptive feature of the control system.

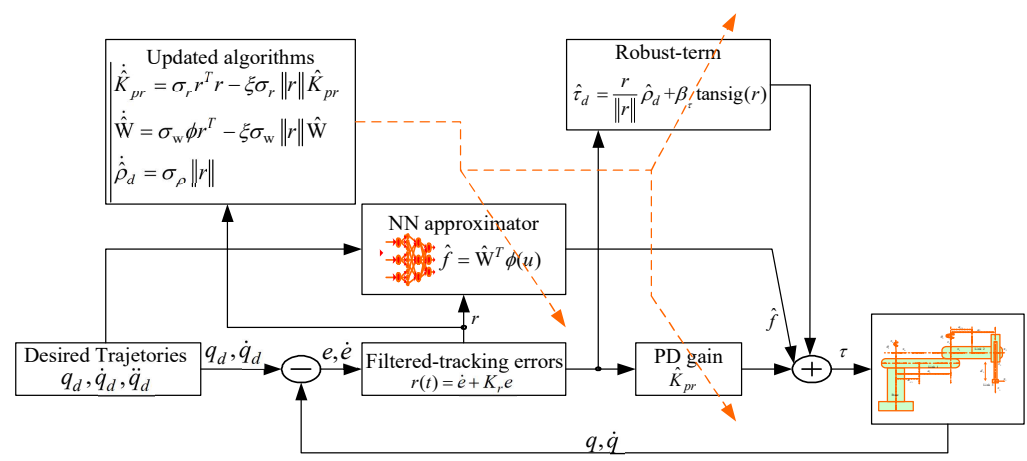

Figure 1: The proposed control system

\section{ADAPTIVE CONTROL ALORITHM}

Our goal is to design an adaptive PD control law to guarantee the position tracking control for the RM system, $q(t) \rightarrow q_{d}(t)$ as time tends to infinity. The structure of the proposed controllers is drawn on the Fig. 2. In order to guarantee the design procedures, we will consider the following assumptions as:

Assumption 1: $\left\|\mathrm{W}^{*}\right\| \leq b_{\mathrm{w}},\|\varepsilon\| \leq b_{\varepsilon}$, where $b_{\mathrm{w}}, b_{\varepsilon}$ are positive real values.

Assumption 2: $\left\|\mathrm{K}_{p r}\right\| \leq b_{\mathrm{k}},\|d\| \leq b_{d}$, where $b_{k}, b_{d}$ are positive real values.

\subsection{Adaptive controller design}

From the aforementioned analysis, in order to solve the drawbacks in the traditional PD-filtered error controller (5), the proposed control input is considered as the following form:

$$
\tau=\hat{f}+\hat{K}_{p r} r+\hat{\tau}_{d}
$$

where $\hat{f}$ is the NN approximation function for the unknown dynamics of the RM control system, $f$ in the Equation (5). $\hat{K}_{p r}$ is the self-updated parameter of for the PD gain. $\hat{\tau}_{d}$ is proposed as the robust term that is used for relaxing the approximating errors and uncertain disturbances in the control process.

By applying the proposed control law (11) into the RM system (1), the RM closed-loop control system may be considered as:

$$
M \dot{r}=-C r+f+d-\hat{f}-\hat{K}_{p r} r-\hat{\tau}_{d}=-C r+\tilde{f}-\hat{K}_{p r} r+d-\hat{\tau}_{d}
$$


Based on the results in (10) and (12), the RM closed-loop control system can be rewritten as:

$$
M \dot{r}=-C r+\tilde{\mathrm{W}}^{T} \phi+\tilde{K}_{p r} r+\varepsilon-K_{p r} r+d-\hat{\tau}_{d}
$$

where $\tilde{K}_{p r} \equiv K_{p r}-\hat{K}_{p r}, K_{p r}$ is considered as the optimal values of the PD gains. According to the Assumptions 1 and 2, by analyzing (13), the following inequality can be obtained as:

$$
\|\varepsilon+d\|+\xi \frac{b_{\mathrm{w}}^{2}}{4}+\xi \frac{b_{k}^{2}}{4} \leq \rho_{d}
$$

where $\xi$ is a positive constant, $\rho_{d}$ is the uncertain bound of $\|\varepsilon+d\|+\xi \frac{b_{\mathrm{w}}^{2}}{2}+\xi \frac{b_{k}^{2}}{2}$. From this result, if the robust-term $\hat{\tau}_{d}$ can eliminate the uncertain part $(\varepsilon+d)$ then the tracking performance of the proposed controller may be improved. Therefore, with the result in (14), the robust-term is proposed as the following form:

$$
\hat{\tau}_{d}=\frac{r}{\|r\|} \hat{\rho}_{d}+\beta_{\tau} \operatorname{tansig}(r)
$$

where $\hat{\rho}_{d}$ is an online estimated value of the $\rho_{d}, \beta_{\tau}$ is a positive constant. The $\operatorname{tansig}(r)$ is the hyperbolic tangent sigmoid function, as shown in the Fig. 3 , that is defined as the following form:

$$
\operatorname{tansig}(r)=\frac{2}{\left(1+e^{-2 r}\right)}-1
$$

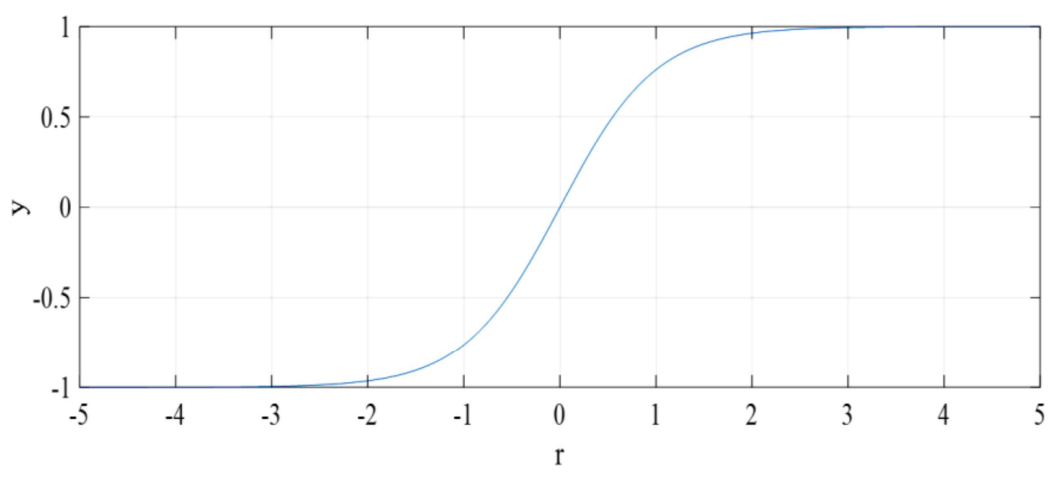

Figure 3: The shape of tansig function

In this research, we will design the adaptive online learning algorithms for the PD gains, the NN parameters and the robust-term parameter. By using the Lyapunov stability theorem [18], the adaptive online learning algorithms for the proposed controller are designed as follows:

\subsection{Stability analysis}

Theorem: By considering the RM dynamics in (1), all the Assumptions hold. If the control laws for the position tracking control and the robust-term are designed as (11) and (15), and the updating algorithms for the RM control system based on the NN are designed as (17), then, the parameters of the NN approximator are bounded, the filtered tracking errors converge to zero, and the stability of the proposed control system is guaranteed.

Proof:

Consider the Lyapunov candidate function as the following form:

$$
V\left(r(t), \tilde{\mathrm{W}}, \tilde{K}_{p r}, \tilde{\rho}_{d}\right)=\frac{1}{2} r^{T} M r+\frac{1}{2} \operatorname{tr}\left(\tilde{\mathrm{W}}^{T} \sigma_{\mathrm{W}}^{-1} \tilde{\mathrm{W}}\right)+\frac{1}{2} \operatorname{tr}\left(\tilde{K}_{p r}^{T} \sigma_{k}^{-1} \tilde{K}_{p r}\right)+\frac{1}{2} \tilde{\rho}_{d} \sigma_{\rho}^{-1} \tilde{\rho}_{d}
$$

where $\tilde{\rho}_{d}=\rho_{d}-\hat{\rho}_{d}$. By differentiating (18) with respect to time, we have: 


$$
\dot{V}=r^{T} M \dot{r}+\frac{1}{2} r^{T} \dot{M} r-\operatorname{tr}\left(\tilde{\mathrm{W}}^{T} \sigma_{\mathrm{W}}^{-1} \dot{\hat{\mathrm{W}}}\right)-\operatorname{tr}\left(\tilde{K}_{p r}^{T} \sigma_{k}^{-1} \dot{\hat{K}}_{p r}\right)-\tilde{\rho}_{d} \sigma_{\rho}^{-1} \dot{\hat{\rho}}_{d}
$$

By substituting (13) into (19), based on the Properties 1 and 2, the following result can be achieved as follows:

$$
\dot{V}=-r^{T} K_{p r} r+r^{T} \tilde{\mathrm{W}}^{T} \phi+r^{T} \tilde{K}_{p r} r+r^{T}(\varepsilon+d)-r^{T} \hat{\tau}_{d}-\operatorname{tr}\left(\tilde{\mathrm{W}}^{T} \sigma_{\mathrm{W}}^{-1} \dot{\hat{\mathrm{W}}}\right)-\operatorname{tr}\left(\tilde{K}_{p r}^{T} \sigma_{k}^{-1} \dot{\hat{K}}_{p r}\right)-\tilde{\rho}_{d}\|r\|
$$

If the adaptive online updating laws for the proposed controller are chosen as (17), then (20) is described as:

$$
\begin{aligned}
\dot{V} & =-r^{T} K_{p r} r+r^{T} \tilde{\mathrm{W}}^{T} \phi+r^{T} \tilde{K}_{p r} r+r^{T}(\varepsilon+d)-r^{T} \hat{\tau}_{d} \\
& -\operatorname{tr}\left(\tilde{\mathrm{W}}^{T} \sigma_{\mathrm{W}}^{-1}\left(\sigma_{\mathrm{w}} \phi r^{T}-\xi \sigma_{\mathrm{w}}\|r\| \mathrm{W}\right)\right)-\operatorname{tr}\left(\tilde{K}_{p r}^{T} \sigma_{k}^{-1}\left(\sigma_{r} r^{T} r-\xi \sigma_{r}\|r\| \hat{K}_{p r}\right)\right)-\tilde{\rho}_{d}\|r\| \\
& =-r^{T} K_{p r} r+r^{T}(\varepsilon+d)-r^{T} \hat{\tau}_{d}+\xi\|r\| \operatorname{tr}\left(\tilde{\mathrm{W}}^{T} \hat{\mathrm{W}}\right)+\xi\|r\| \operatorname{tr}\left(\tilde{K}_{p r}^{T} \hat{K}_{p r}\right)-\tilde{\rho}_{d}\|r\|
\end{aligned}
$$

According to the following results [1] as $\operatorname{tr}\left(\tilde{\mathrm{W}}^{T} \hat{\mathrm{W}}\right) \leq \mathrm{b}_{\mathrm{w}}\|\tilde{\mathrm{W}}\|-\|\tilde{\mathrm{W}}\|^{2}, \operatorname{tr}\left(\tilde{K}_{p r}^{T} \hat{K}_{p r}\right) \leq \mathrm{b}_{k}\left\|\tilde{\mathrm{K}}_{p r}\right\|-\left\|\tilde{\mathrm{K}}_{p r}\right\|^{2}$, from (21), we may obtain the following inequality as:

$$
\dot{V} \leq-r^{T} K_{p r} r+r^{T}(\varepsilon+d)-r^{T} \hat{\tau}_{d}-\tilde{\rho}_{d}\|r\|+\xi\|r\|\left(\mathrm{b}_{\mathrm{w}}\|\tilde{\mathrm{W}}\|-\|\tilde{\mathrm{W}}\|^{2}\right)+\xi\|r\|\left(\mathrm{b}_{k}\left\|\tilde{\mathrm{K}}_{p r}\right\|-\left\|\tilde{\mathrm{K}}_{p r}\right\|^{2}\right)
$$

By substituting (14) into (22), yields:

$$
\begin{aligned}
\dot{V} & \leq-r^{T} K_{p r} r-r^{T} \hat{\tau}_{d}-\tilde{\rho}_{d}\|r\|+\|r\| \rho_{d}-\xi\|r\|\left(\frac{b_{\mathrm{w}}}{2}-\|\tilde{\mathrm{W}}\|\right)^{2}-\xi\|r\|\left(\frac{b_{k}^{2}}{4}-\left\|\tilde{\mathrm{K}}_{p r}\right\|\right)^{2} \\
& \leq-r^{T} K_{p r} r-r^{T} \hat{\tau}_{d}-\tilde{\rho}_{d}\|r\|+\| r \mid \rho_{d}
\end{aligned}
$$

By applying the robust-term (15) into (23), it is concluded that:

$$
\begin{aligned}
\dot{V} & \leq-r^{T} K_{p r} r-r^{T}\left(\frac{r}{\|r\|} \hat{\rho}_{d}+\beta_{\tau} \operatorname{tansig}(r)\right)-\tilde{\rho}_{d}\|r\|+\|r\| \rho_{d} \\
& \leq-r^{T} K_{p r} r-r^{T} \beta_{\tau} \operatorname{tansig}(r)-\|r\| \hat{\rho}_{d}-\tilde{\rho}_{d}\|r\|+\|r\| \rho_{d} \\
& \leq-r^{T} K_{p r} r-r^{T} \beta_{\tau} \operatorname{tansig}(r) \\
& \leq-r^{T} K_{p r} r
\end{aligned}
$$

From the result in (24), the $\dot{V}\left(r(t), \tilde{\mathrm{W}}, \tilde{K}_{p r}, \tilde{\rho}_{d}\right)$ is a negative semi-definite function, thus, $V\left(r(t), \tilde{\mathrm{W}}, \tilde{K}_{p r}, \tilde{\rho}_{d}\right) \leq V\left(r(0), \tilde{\mathrm{W}}, \tilde{K}_{p r}, \tilde{\rho}_{d}\right)$. If $r(t), \tilde{\mathrm{W}}, \tilde{K}_{p r}, \tilde{\rho}_{d}$ are bounded at initial time $(t=0)$, they will stay this bounded state for $(t \geq 0)$. And also, $\hat{\mathrm{W}}, \hat{K}_{p r}, \hat{\rho}_{d}$ are bounded for $(t \geq 0)$. Defining that $\Pi=-r^{T} K_{p r} r$, we have $\Pi \leq-\dot{V}$. By integrating $\Pi(t)$ with respect to time, yields:

$$
\int_{0}^{t} \Pi(\varsigma) d \varsigma \leq V\left(r(0), \tilde{\mathrm{W}}, \tilde{K}_{p r}, \tilde{\rho}_{d}\right)-V\left(r(t), \tilde{\mathrm{W}}, \tilde{K}_{p r}, \tilde{\rho}_{d}\right)
$$

With $V(0)$ is a bounded function, and the $V(t)$ is a non-increasing and bounded function, the following result can be obtained as:

$$
\left.\lim _{t \rightarrow \infty} \int_{0}^{t} \Pi(\varsigma) d \varsigma\right)<\infty
$$

So, by applying the Barbalat's Lemma [18] with $\dot{\Pi}(t)$ is bounded, it shows that $\left.\lim _{t \rightarrow \infty} \int_{0}^{t} \Pi(\varsigma) d \varsigma\right)=0$ and the filtered-tracking errors will converge $r(t)$ to zero as time tends to infinity. Here, based on this result, we can see that the control input is bounded from (11). Therefore, we can conclude that the stability and robustness of the proposed control system are guaranteed. In addition, when the filtered - tracking errors converge to zero lead to the tracking errors will converge to zero as time tends to infinity. 


\section{NUMERICAL SIMULATION RESULTS}

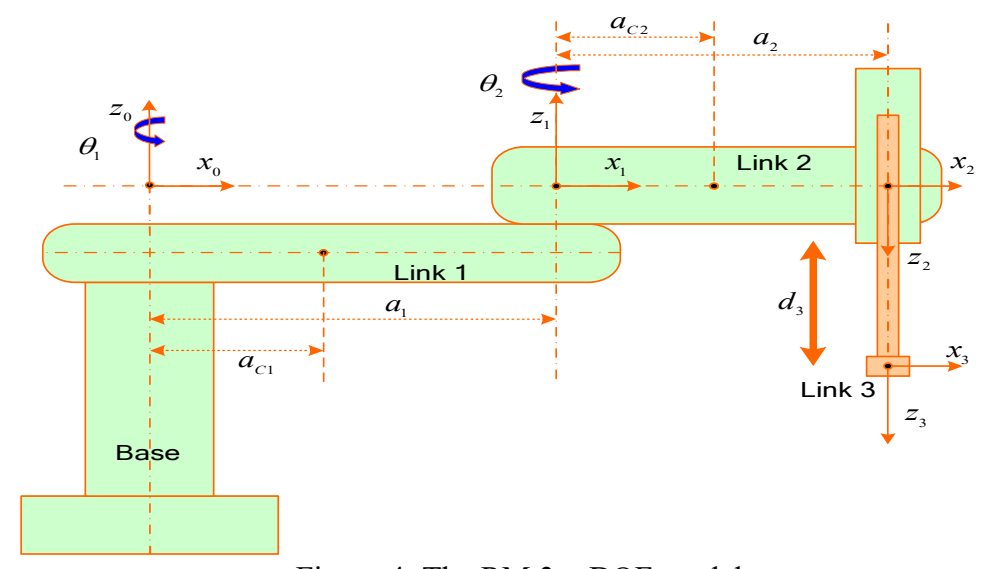

Figure 4: The RM 3 - DOF model

In this research, the RM 3 - DOF model, as shown in the Fig. 4, will be used in the simulation process to prove the effectiveness of the proposed control method. The model of RM is constructed in the Lagrange equations form, with its dynamics is given as in [1]. In order to exhibit the superior control performance of the proposed control scheme (APDNN), the traditional PD controller, the conventional PDNN strategy in [1] will be examined. The PD controller input is considered as follows: $\tau=K_{p} e(t)+K_{d} \dot{e}(t)$. For the comparison of control results are favorable, the PDNN schemes in [1] are modified according to the proposed controller form. The control parameters of the PD-NN and proposed controller in this simulation work can be summarized in the Table 1 .

We note that, all the aforementioned control constant parameters are chosen to achieve the best transient control performance in the numerical simulation process by considering the requirement of the stability and the possible operating conditions. For recording the respective control performances, the mean square error (MSE) of the position tracking response is defined as: $M S E=\frac{1}{T} \sum_{k=1}^{T}\left[q(k)-q_{d}(k)\right]^{2}$, where $\mathrm{T}$ is the total sampling instants. Based on this definition, the normalized MSE (NMSE) value of the position tracking response by using a per-unit value with $1 \mathrm{rad}$ is used to examine the control performance.

Table 1: Control parameters

\begin{tabular}{|c|c|c|c|}
\hline \multirow{2}{*}{$\begin{array}{c}\text { Control } \\
\text { parameters }\end{array}$} & \multicolumn{3}{|c|}{ Control methods } \\
\hline & $\begin{array}{l}\text { The proposed controller } \\
\text { (APDNN) }\end{array}$ & The PDNN controller [1] & PD controller \\
\hline$K_{r}$ & $\operatorname{diag}(10)$ & $\operatorname{diag}(10)$ & $K_{p}=\operatorname{diag}(100,90,50)$ \\
\hline$K_{p r}$ & updated & $\operatorname{diag}(100,70,50)$ & $K_{d}=\operatorname{diag}(1500,1000,800)$ \\
\hline$\sigma_{\mathrm{r}}$ & $\operatorname{diag}(20000)$ & - & - \\
\hline$\sigma_{\mathrm{w}}$ & $\operatorname{diag}(10000)$ & $\operatorname{diag}(10000)$ & - \\
\hline$\sigma_{p}$ & $\operatorname{diag}(50)$ & $\operatorname{diag}(50)$ & - \\
\hline$\xi$ & $\operatorname{diag}(0.001)$ & $\operatorname{diag}(0.001)$ & - \\
\hline
\end{tabular}

The desired joint positions of the RM are defined as $q_{d}=[0.5 \sin (1.5 t), 0.5 \sin (2 t), 0.1 \sin (1.5 t)](\mathrm{rad})$, the friction term is considered as: $F(\dot{q})=\left[0.1 \dot{q}_{1}+0.2 \operatorname{sign}\left(\dot{q}_{1}\right) ; 0.2 \dot{q}_{2}+0.2 \operatorname{sign}\left(\dot{q}_{2}\right) ; 0.1 \dot{q}_{3}+0.2 \operatorname{sign}\left(\dot{q}_{3}\right)\right]$, the disturbance term is $\tau_{d}=[0.2 \sin (2 t) ; 0.2 \sin (2 t) ; 0.2 \sin (2 t)]$. The simulation process is carried out using the Matlab software with sample time is $0.001 \mathrm{~s}$. The simulation process is implemented as follows. When simulation time is $2.5 \mathrm{~s}, \tau_{d}=20[\sin (15 t) ; \sin (20 t) ; \sin (15 t)]$, the tip loads will add to the links 2 and 3 of the $\mathrm{RM}$ system (2 kg and $1.5 \mathrm{~kg}$ on links 1 and 2). At simulation time is $5 \mathrm{~s}$, the friction term will change to $F(\dot{q})=\left[\dot{q}_{1}+8 \operatorname{sign}\left(\dot{q}_{1}\right) ; \dot{q}_{2}+6 \operatorname{sign}\left(\dot{q}_{2}\right) ; \dot{q}_{3}+4 \operatorname{sign}\left(\dot{q}_{3}\right)\right]$. 

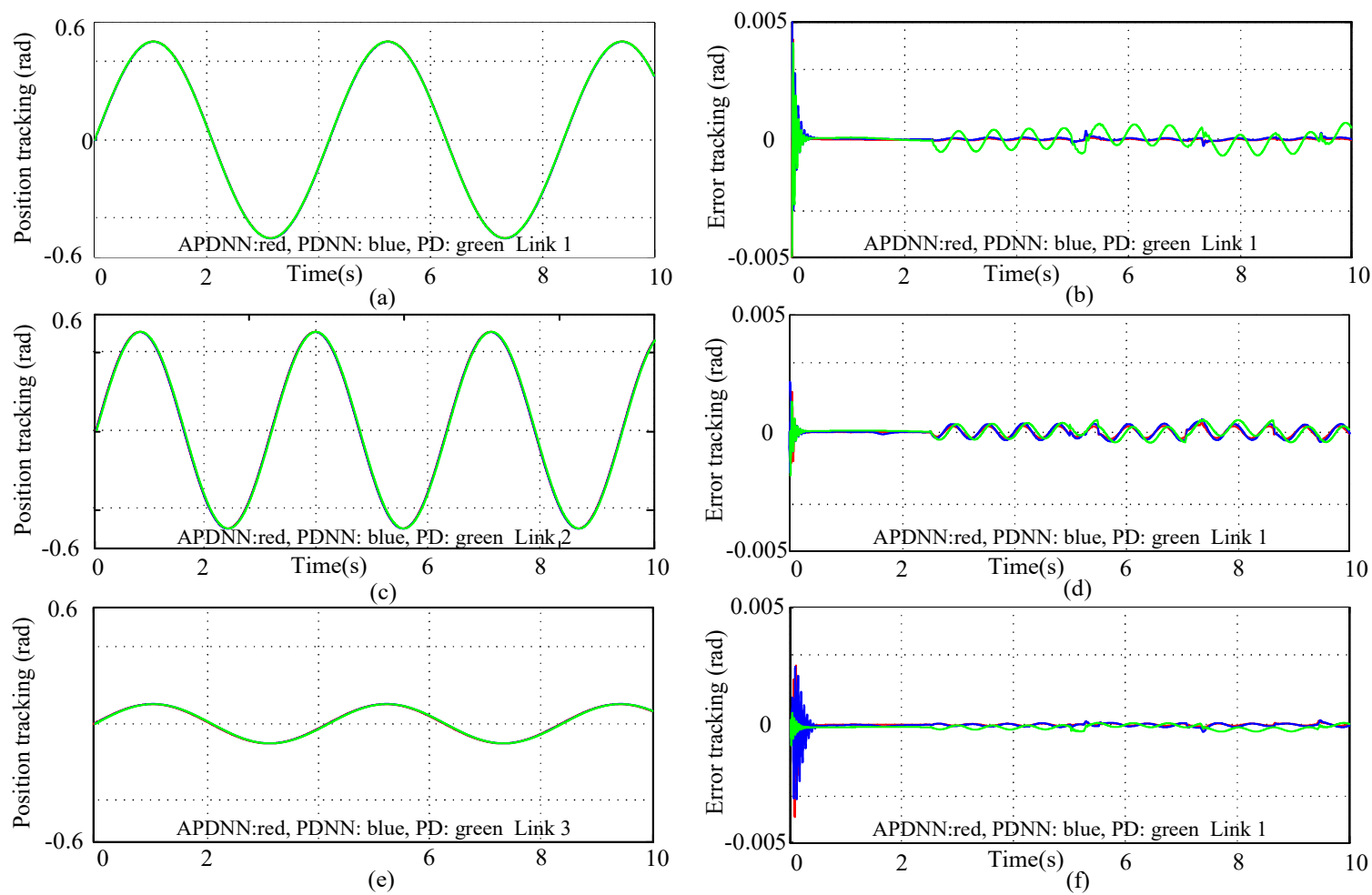

Figure 5: The tracking positions and errors performance.

Table 2: Simulation performance comparisons

\begin{tabular}{|l|l|l|l|}
\hline NMSE $\left(\mathrm{x} 10^{-4} \mathrm{rad}\right)$ & Link1 & Link2 & Link3 \\
\hline Adaptive PDNN & 3.014 & 1.802 & 1.979 \\
\hline PDNN & 3.871 & 2.275 & 2.546 \\
\hline PD & 4.281 & 2.430 & 3.375 \\
\hline
\end{tabular}
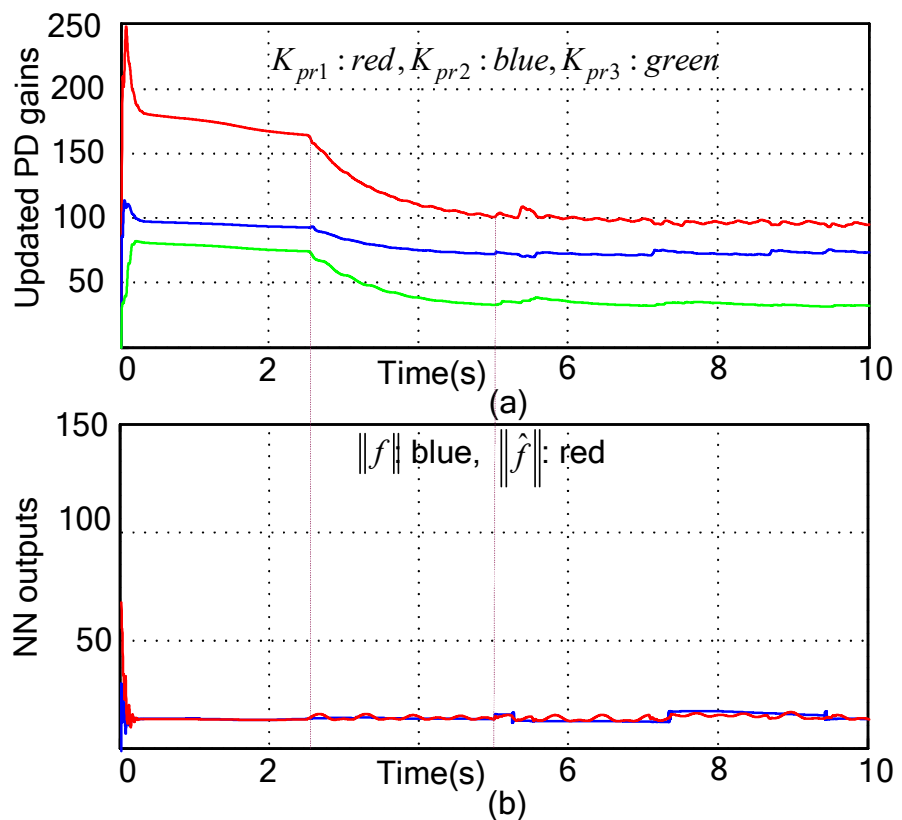

Figure 6: The updated parameters of the PD gains and NN outputs.

The Fig. $5 \mathrm{a}-\mathrm{f}$ show the simulation results of the tracking positions and errors performance. From the 
simulation results in the Fig 5. a, c and e, we can see the positions of the RM, for the proposed control method, the PDNN method and PD method, are well track to the desired position. By observing the tracking errors performance in the Fig. $5 \mathrm{~b}, \mathrm{~d}$ and f, we can easily realize that, the proposed control scheme achieve better tracking error - performances than those of the PDNN [1] and PD controllers. This is also confirming in the NMSE comparison values in the Table 2. The Fig. $6 \mathrm{a}$ and $\mathrm{b}$ provide the simulation results of the updated parameters of the PD gains and NN outputs. These results first show the effectiveness of the updating algorithms in guaranteeing the adaptation and robustness (boundedness) of control signals off the adaptive PD controller and NN approximator. In the other hand, the PD gain parameters have self-adapted when the control environment conditions changed dramatically. Therefore, the tracking performance of the proposed control method is considered to be better. In the simulation process, the PDNN and PD controllers can obtain the good performances caused by selecting the PD parameters. The choosing procedures for the PD gain parameters are not easy to implement. In this simulation process, by observing the results about adaptive PD parameters of the proposed controller, we have convenient methods to obtain the acceptable optimal PD parameters for the PDNN and PD controllers.
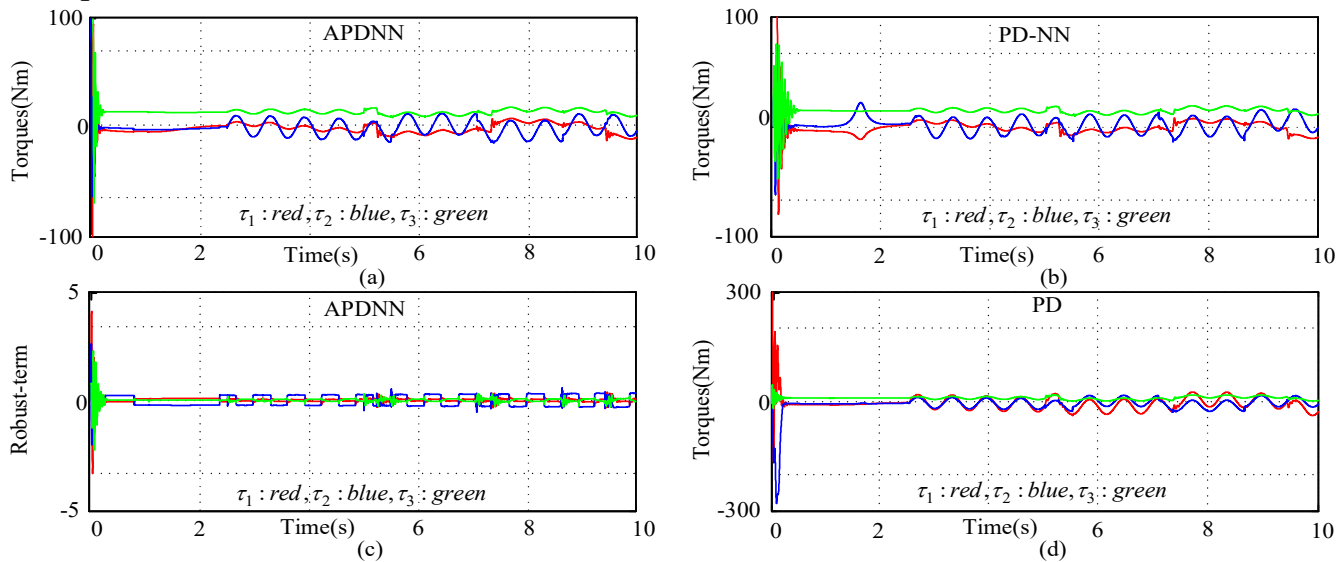

Figure 7: The control inputs.

Based the simulation results in the Fig. $7 \mathrm{c}$, we can see the effectiveness of the robust-term controllers. This controller has provided the compensating values for the main controller and it has guaranteed the boundedness in the control process. And also, follow to the results in the Fig. 7 a, b and d, the control inputs of the proposed controller, the PDNN controller and the PD controller are good. However, the PD control created the higher control inputs at initial time. This can be a drawback in the real-time practical applications. With the aforementioned analysis, when compare the simulation results of the proposed adaptive PDNN, PDNN and PD controllers, we can conclude that the proposed control strategy has not only prove the effectiveness in the adaptation and flexibility, but also guarantee the good performance in tracking position control.

\section{CONCLUSIONS}

In this paper, the novel proposed control system using the adaptive PD technique has been successfully applied for the RM control system. The proposed adaptive PD control scheme, with the highly adaptive self-turning PD gains and uncertainties elimination abilities, has improved the tracking position control, flexibility and robustness for the RM control system. In addition, all the adaptive updating/learning algorithms for the proposed control parameters are online implemented, which are obtained by using the Lyapunov stability theorem. Thus, the stability and adaptability of the proposed control system are guaranteed without considering uncertain parts occupation. The proposed control system can be considered to apply as a good alternative in the existing RM control system.

\section{ACKNOWLEDGMENT}

This work was supported by the Industrial University of Ho Chi Minh City, Vietnam (No. 360/QĐ-ĐHCN, 22/03/2021).

The authors would like to thank the associate editor and the reviewers for their valuable comments. 


\section{REFERENCES}

[1] T. L. Mai, Y. N. Wang and T. Q. Ngo, Adaptive tracking control for robot manipulators using fuzzy wavelet neural networks, International Journal of Robotics and Automation, vol. 30, no. 1, pp. 26-39, 2015.

[2] J. Guan, C. Lin, G. Ji, L. Qian and Y. Zheng, Robust Adaptive Tracking Control for Manipulators Based on a TSK Fuzzy Cerebellar Model Articulation Controller, IEEE Access, vol. 6, pp. 1670-1679, 2018.

[3] S. Zhang, Y. Dong, Y. Ouyang, Z. Yin and K. Peng, Adaptive Neural Control for Robotic Manipulators With Output Constraints and Uncertainties, IEEE Transactions on Neural Networks and Learning Systems, vol. 29, no. 11, pp. 5554-5564, Nov. 2018.

[4] C. Yang, Y. Jiang, W. He, J. Na, Z. Li and B. Xu, Adaptive Parameter Estimation and Control Design for Robot Manipulators With Finite-Time Convergence, IEEE Transactions on Industrial Electronics, vol. 65, no. 10, pp. 81128123, Oct. 2018.

[5] W. He, Z. Yan, Y. Sun, Y. Ou and C. Sun, Neural-Learning-Based Control for a Constrained Robotic Manipulator With Flexible Joints, IEEE Transactions on Neural Networks and Learning Systems, vol. 29, no. 12, pp. 5993-6003, Dec. 2018.

[6] L. Zhang, Y. Wang, Y. Hou and H. Li, Fixed-Time Sliding Mode Control for Uncertain Robot Manipulators, IEEE Access, vol. 7, pp. 149750-149763, 2019.

[7] Q. Zhou, S. Zhao, H. Li, R. Lu and C. Wu, Adaptive Neural Network Tracking Control for Robotic Manipulators With Dead Zone, IEEE Transactions on Neural Networks and Learning Systems, vol. 30, no. 12, pp. 3611-3620, Dec. 2019.

[8] B. Xiao and S. Yin, Exponential Tracking Control of Robotic Manipulators With Uncertain Dynamics and Kinematics, IEEE Transactions on Industrial Informatics, vol. 15, no. 2, pp. 689-698, Feb. 2019.

[9] M. Van, M. Mavrovouniotis and S. S. Ge, An Adaptive Backstepping Nonsingular Fast Terminal Sliding Mode Control for Robust Fault Tolerant Control of Robot Manipulators, IEEE Transactions on Systems, Man, and Cybernetics: Systems, vol. 49, no. 7, pp. 1448-1458, July 2019.

[10] V. Narayanan, S. Jagannathan and K. Ramkumar, Event-Sampled Output Feedback Control of Robot Manipulators Using Neural Networks, IEEE Transactions on Neural Networks and Learning Systems, vol. 30, no. 6, pp. 1651-1658, June 2019.

[11] E. Arefinia, H. A. Talebi and A. Doustmohammadi, A Robust Adaptive Model Reference Impedance Control of a Robotic Manipulator With Actuator Saturation, IEEE Transactions on Systems, Man, and Cybernetics: Systems, vol. 50, no. 2, pp. 409-420, Feb. 2020.

[12] A. Perrusquía, J. A. Flores-Campos and C. R. Torres-San-Miguel, A Novel Tuning Method of PD With Gravity Compensation Controller for Robot Manipulators, IEEE Access, vol. 8, pp. 114773-114783, 2020.

[13] Y. Su, Comments on “A New Adaptive Sliding-Mode Control Scheme for Application to Robot Manipulators”, IEEE Transactions on Industrial Electronics, vol. 67, no. 8, pp. 7116-7120, Aug. 2020.

[14] M. A. Arteaga-Peréz, J. Pliego-Jiménez and J. G. Romero, Experimental Results on the Robust and Adaptive Control of Robot Manipulators Without Velocity Measurements, IEEE Transactions on Control Systems Technology, vol. 28 , no. 6 , pp. 2770-2773, Nov. 2020. 
[15] L. Dai, Y. Yu, D. -H. Zhai, T. Huang and Y. Xia, Robust Model Predictive Tracking Control for Robot Manipulators With Disturbances, IEEE Transactions on Industrial Electronics, vol. 68, no. 5, pp. 4288-4297, May 2021.

[16] J. Zhai and G. Xu, A Novel Non-Singular Terminal Sliding Mode Trajectory Tracking Control for Robotic Manipulators, IEEE Transactions on Circuits and Systems II: Express Briefs, vol. 68, no. 1, pp. 391-395, Jan. 2021.

[17] F. L. Lewis, K. Liu and A. Yesildirek, Neural net robot controller with guaranteed tracking performance, IEEE Transactions on Neural Networks, vol. 6, no. 3, pp. 703-713, 1996.

[18] J. J. E. Slotine, W. Li, Applied Nonlinear Control, Englewood Cliffs, NJ: Prentice-Hall, 1991.

\section{MộT PHƯƠNG PHÁP ĐIỀU KHIỂN VI PHÂN-TỶ LỆ THÍCH NGHI CHO HỆ ROBOT TAY MÁY}

Tóm tắt. Nghiên cứu này trình bày một phương pháp điều khiển cải tiến cho hệ robot tay máy dựa trên kỹ thuật vi phân-tỷ lệ và mạng nơ ron. Ở chiến lượt được đề xuất này, bộ điều khiển vi phân tỷ lệ dựa trên kỹ thuật sai số bám được lọc đã được hiệu chỉnh để các tham số độ lợi vi phân-tỷ lệ được cập nhật thích nghi. Tương tự với các phương pháp điều khiển thông minh truyền thống, bộ xấp xỉ nơ ron được ứng dụng để giải phóng các đặc tính động chưa biết của hệ điều khiển robot. Hơn nữa bộ điều khiển bền vững dạng bộ bù cũng được xem xét để loại trừ các lỗi xấp xỉ và các nhiễu chưa biết không thể tránh khỏi của hệ thống điều khiển. Bằng việc sử dụng định lý ổn định Lyapunov trong quá trình thiết kế bộ điều khiển đề xuất, việc điều khiển bám và sự ổn định được đảm bảo. Các kết quả mô phỏng sẽ cung cấp các chứng cứ rõ ràng để chứng tỏ sự hiệu quả của đề xuất.

Từ khóa. Robot tay máy, điều khiển PD/PID, điều khiển thích nghi, điều khiển thông minh.

Received on: 12/04/2021

Accepted on: 30/06/2021 